\title{
Intensity-modulated radiation therapy or volumetric-modulated arc therapy to reduce alopecia, xerostomia, and otitis after whole brain radiation therapy for brain metastases: a planning analysis
}

\author{
Brandon R. Mancini • J. Ben Wilkinson • \\ Leonard H. Kim • Simona F. Shaitelman • Di Yan • \\ Dan Ionascu • Inga S. Grills
}

Received: 21 September 2012 / Accepted: 11 January 2013 /Published online: 31 January 2013

(C) Springer-Verlag Berlin Heidelberg 2013

\begin{abstract}
Objective The purpose of this study is to investigate the potential for reducing dose to the scalp, parotid glands, and external auditory canals using intensity-modulated radiation therapy (IMRT) or volumetrically modulated arc therapy (VMAT) to deliver whole brain radiation therapy (WBRT).

Methods Nine patients with brain metastases previously treated with standard opposed lateral (OL) WBRT were studied. Four new plans were created for each patient: (1) four-beam IMRT, (2) seven-beam IMRT, (3) 13-beam IMRT, and (4) VMAT (single arc technique). All plans were optimized to achieve equivalent planning target volume (PTV) coverage while minimizing dose to the scalp, parotid gland, and external ear. Each of the plans was then
\end{abstract}

Prior presentations: Poster session presented at the 52nd Annual Meeting of the American Society of Radiation Oncology (ASTRO); 31 Oct 31-4 Nov 4, 2010; San Diego, CA

B. R. Mancini

Department of Therapeutic Radiology, Yale University,

New Haven, CT, USA

J. B. Wilkinson • D. Yan • D. Ionascu • I. S. Grills ( $\square)$

Department of Radiation Oncology, Beaumont Cancer Institute,

Oakland University William Beaumont School of Medicine,

Royal Oak, MI, USA

e-mail: igrills@beaumont.edu

L. H. Kim

Department of Radiation Oncology,

Robert Wood Johnson Medical School, New Brunswick, NJ, USA

S. F. Shaitelman

Department of Radiation Oncology, University of Texas M.D.

Anderson Cancer Center, Houston, TX, USA dosimetrically compared via multiple dose-volume histogram (DVH) parameters.

Results All IMRT and VMAT plans significantly reduced dose to the scalp, ears, and parotid glands without increasing dose to other critical structures. Improved scalp sparing for low, intermediate, and high doses $\left(\mathrm{V}_{5}, \mathrm{~V}_{10}, \mathrm{~V}_{20}\right.$, and $\left.\mathrm{V}_{30}\right)$, was achieved with the 4- and 13-beam IMRT plans. The 7field plan reduced intermediate and high scalp doses $\left(\mathrm{V}_{10}\right.$, $\mathrm{V}_{20}$, and $\left.\mathrm{V}_{30}\right)$ while only high doses $\left(\mathrm{V}_{20}\right.$ and $\left.\mathrm{V}_{30}\right)$ were reduced with the VMAT plan. Depending on the DVH parameter, IMRT/VMAT reduced scalp dose between $10 \%$ and $70 \%$, parotid gland dose by $3 \%$ and $31 \%$, and external auditory canal dose by $2 \%$ and $96 \%$.

Conclusion IMRT or VMAT is able to reduce dose to the scalp, parotid glands, and external auditory canal without sacrificing PTV coverage when compared to a standard OL treatment plan. Such improvement is promising and may decrease non-cognitive WBRT toxicities (e.g., alopecia, xerostomia, otitis), which could improve quality of life for patients receiving WBRT. Future planning will attempt to reduce cochlear dose to avoid hearing loss but will likely require PTV modification.

Keywords Brain metastases · IMRT - Whole brain radiation therapy $\cdot$ Alopecia $\cdot$ Radiation side effects

\section{Introduction}

Brain metastases are ten times more common than intracranial tumors with an estimated incidence between 170,000 and 200,000 cases per year in the USA [1]. In fact, it is estimated that between $20 \%$ and $30 \%$ of cancer patients 
have intracranial spread of their disease at the time of death [2]. Median survival for patients treated with supportive care is between 1 and 2 months and, although metastatic disease can cause disruptions in the blood-brain barrier, systemic therapy is generally not effective for intracranial disease. Because of this, the most common treatment option for patients with three or more brain metastases is whole brain radiotherapy (WBRT), which has the potential of extending the median survival from 1 month to 4 months, or longer depending on the primary histology $[3,4]$.

Although effective at decreasing the incidence of new intracranial disease and preventing death from a neurologic cause [5], this treatment can create significant acute and chronic side effects, which may affect patient quality of life $[6,7]$. Common side effects after WBRT include temporary and permanent alopecia, xerostomia, otitis, hearing loss, and neurocognitive decline. Specifically, Lawenda et al. noted a dose-response relationship for permanent alopecia after cranial irradiation [8]. In the past, many WBRT dose/fractionation schedules have been investigated, but none have shown superior survival or significant reduction of untoward side effects following treatment $[8,9]$. Recently, there have been attempts to decrease hippocampal dose with the intent of decreasing and/or eliminating neurocognitive decline associated with WBRT $[10,11]$. In the present study, we examine whether intensity-modulated (IMRT) or volumetricmodulated arc (VMAT) treatment planning techniques have the capacity to meaningfully reduce dose to the scalp, parotid glands, and external auditory canals without compromising planning tumor volume (PTV) coverage.

\section{Methods}

With approval from our institutional review board, we studied nine patients who had previously been diagnosed with brain metastases and were treated at our institution with standard, two-beam opposed lateral WBRT. Computed tomography (CT)-based treatment planning images were available for each of these patients, and these data sets were used to repeat the treatment planning process using intensity-modulated and volumetric-modulated arc techniques. Four new plans were created for each patient: (1) fourbeam IMRT (Fig. 1), (2) seven-beam IMRT, (3) 13-beam IMRT, and (4) VMAT (single arc technique) and multiple normal tissue constraints were placed on identified organs at risk within the patient image sets (Table 1). The number and arrangement of beams were selected in order to provide homogeneous dose distribution to the PTV while also understanding clinical applicability of devised plans. Normal tissue structures were manually contoured by a single individual using Pinnacle 3 treatment planning software, version $8.1 \mathrm{~s}$. These structures included the parotid glands, external ears, internal auditory canals, cochlea, eyes, lenses, optic nerves, brain (to the bottom of the medulla), skull, skin, and soft tissue of the scalp (defined as skin minus $0.5-\mathrm{cm}$ depth). All four created plans were then compared to the original opposed lateral plan with respect to multiple normal tissue dose parameters. Normal tissue constraints were determined using the As Low As Reasonably Achievable principle. If normal tissue constraints (Table 1) were not able to be satisfied due to the fact that decreasing tissue dose would sacrifice PTV coverage, we ensured all critical structures received the same or lower dose than they did in the opposed lateral plan. IMRT plans included a mixture of coplanar and vertex beams set to avoid entry or exit through eyes, ears, and mouth, when possible (Table 2). The PTV was defined as whole brain plus a $0.5-\mathrm{cm}$ uniform $3 \mathrm{D}$ expansion. The prescribed dose was 37.5 Gy in 15 fractions; volumetrically for the IMRT and VMAT plans and to mid-plane for the opposed lateral plan. All plans were optimized to achieve equivalent uniform PTV coverage while maintaining an equal or lower dose to lenses, eyes, optic nerves, brainstem, internal auditory canals, and bilateral cochlea as compared to the opposed lateral technique. The IMRT cost function was adjusted manually in each case, as needed, to meet treatment planning objectives and weighted such that priority was given to PTV coverage followed by objectives to reduce dose to the scalp, parotid glands, and external auditory canals. Cochlear dose could not be reduced in this setting due to its inclusion/close proximity to the PTV (brain+ $0.5 \mathrm{~cm}$ ). Once all of the new plans were complete, multiple dose-volume histogram (DVH) parameters for the PTV and organs at risk were compared for statistical and clinically meaningful reductions in dose and PTV coverage. Parameters assessed included mean dose, minimum dose, maximum dose, $\mathrm{V}_{5}, \mathrm{~V}_{10}, \mathrm{~V}_{15}, \mathrm{~V}_{20}$, and $\mathrm{V}_{30}$ for each region of interest.

\section{Results}

All intensity-modulated and volumetric-modulated arc plans for each of the nine patients reduced dose to the scalp, ears, and parotid glands without increasing dose to the lenses, eyes, optic nerves, brainstem, internal auditory canals, or bilateral cochlea (Table 3). The mean parotid gland dose was reduced in all enhanced plans by at least $60 \%$ with the greatest reduction being realized by the 13 -field IMRT plan. Similarly, all IMRT/VMAT plans reduced the mean dose to the external auditory canal and pinna by at least $15 \%$ with the lowest dose achieved to these structures observed in the 13-field IMRT plans (mean dose reduced by $41.5 \%$, bilaterally).

Regarding scalp dose, improved sparing was seen for low, intermediate, and high doses $\left(\mathrm{V}_{5}, \mathrm{~V}_{10}, \mathrm{~V}_{20}\right.$, and $\left.\mathrm{V}_{30}\right)$ with the four- and 13-beam IMRT plans. The seven-beam IMRT plan improved intermediate and high doses $\left(\mathrm{V}_{10}, \mathrm{~V}_{20}\right.$, 
Fig. 1 Four-Beam IMRT setup

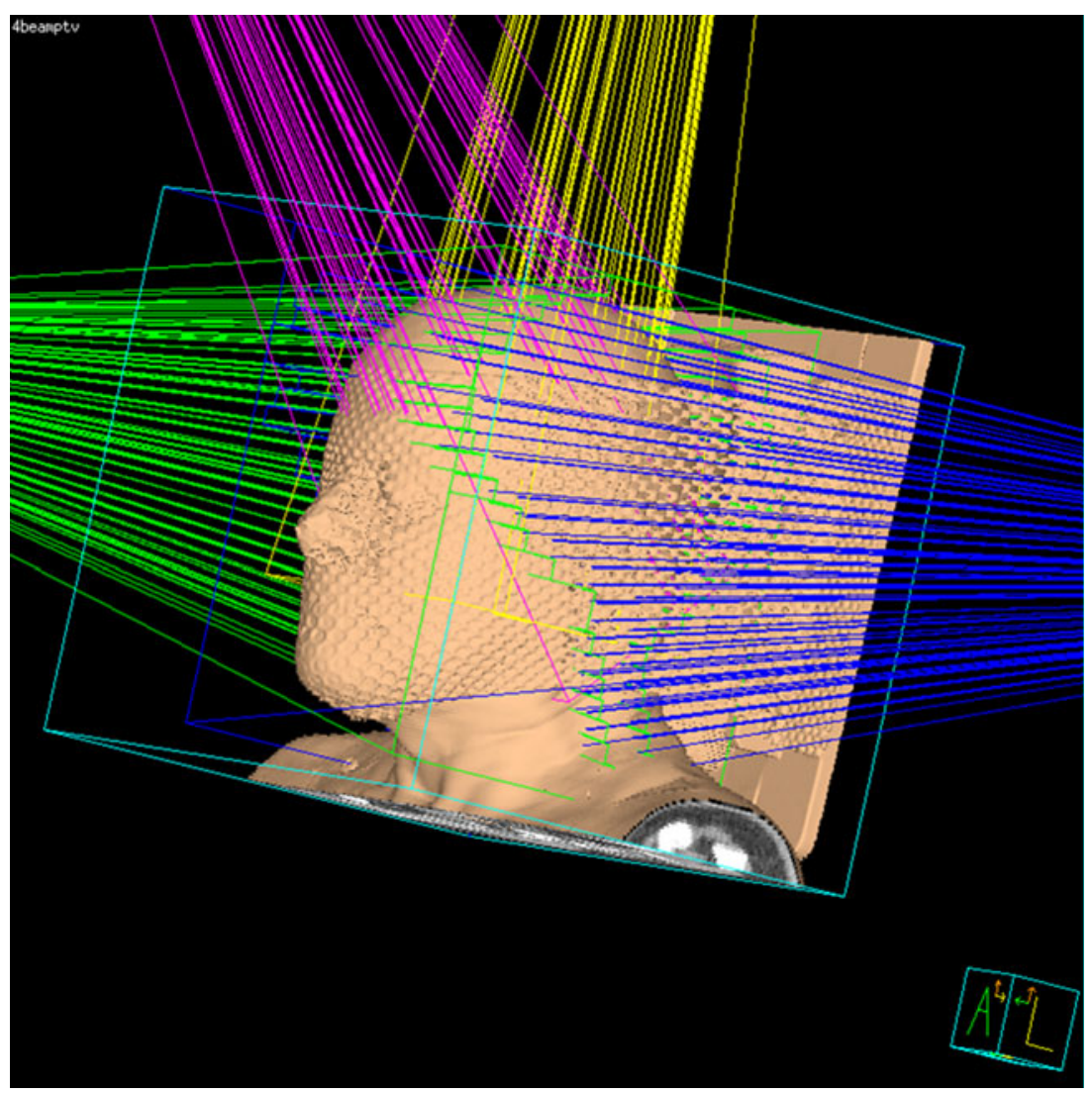

and $\left.\mathrm{V}_{30}\right)$ while only high doses $\left(\mathrm{V}_{20}\right.$ and $\left.\mathrm{V}_{30}\right)$ were reduced in VMAT plans (Table 4). Compared with the OL technique, the four-field IMRT plan reduced the mean dose to the scalp the most (20.8\% reduction) followed by the 13 -field $(-19.8 \%)$, seven-field $(-13.8 \%)$, and VMAT $(-4.8 \%)$. Depending on the DVH parameter, IMRT/VMAT reduced scalp dose by 10-70\%, external ear/auditory canal dose by 2-96 \%, and parotid gland dose by 3-31\% (Fig. 2).

Mean dose to the PTV increased with IMRT and VMAT treatment planning techniques. The mean PTV dose ranged from 39 to 40 Gy for IMRT/VMAT plans versus 38 Gy for OL plans, which represented a $2-5 \%$ increase. The

Table 1 Initial normal structure tissue constraints

\begin{tabular}{llll}
\hline Normal tissues to contour & $D_{\text {mean }}$ & $D_{\text {max }}$ & Coverage \\
\hline R/L parotid glands & $20 \mathrm{~Gy}$ & \\
R/L external ear/external & & $15 \mathrm{~Gy}$ & \\
$\quad$ auditory canal & & \\
R/L internal auditory canal & $20 \mathrm{~Gy}$ & \\
R/L cochlea/labyrinth & $20 \mathrm{~Gy}$ & \\
R/L lens & $<10 \mathrm{~Gy}$ & \\
R/L optic nerve & $37.5 \mathrm{~Gy}$ & \\
Entire brain & & $D_{95}=37.5 \mathrm{~Gy}$ \\
(to bottom of medulla) & & \\
\hline
\end{tabular}

$R$ right, $L$ left planning technique that increased the mean PTV dose the most was VMAT with an average increase in mean dose of 2.1 Gy or $5.5 \%$. The maximum dose to the PTV was moderately higher for IMRT/VMAT (46-50 Gy) as compared to the OL technique (42 Gy); however, hard-stop parameters were not put in place to absolutely control max PTV dose. The mean dose to the optic nerve, lens, and cochlea were 27, 3.6, and 33 Gy, respectively and similar across all plans. In addition, dose homogeneity of the PTV was maintained compared

Table 2 IMRT beam arrangement

\begin{tabular}{lcc}
\hline & Couch angle (degree) & Gantry angle (degree) \\
\hline Beam \#1 & 0 & 270 \\
Beam \#2 & 0 & 90 \\
Beam \#3 & 90 & 90 \\
Beam \#4 & 90 & 45 \\
Beam \#5 & 90 & 215 \\
Beam \#6 & 45 & 90 \\
Beam \#7 & 135 & 90 \\
Beam \#8 & 0 & 0 \\
Beam \#9 & 0 & 180 \\
Beam \#10 & 0 & 45 \\
Beam \#11 & 0 & 135 \\
Beam \#12 & 0 & 225 \\
Beam \#13 & 0 & 315 \\
\hline
\end{tabular}


Table 3 Mean dose delivered to contoured organs at risk and PTV (centigray)

$R$ right, $L$ left

\begin{tabular}{lrrrrr}
\hline & 2-Beam OL & 4-Beam IMRT & 7-Beam IMRT & 13-Beam IMRT & VMAT \\
\hline PTV & $3,810.5$ & $3,908.3$ & $3,896.5$ & $3,876.9$ & $4,021.5$ \\
Brain & $3,857.2$ & $3,976.6$ & $3,953.9$ & $3,936.2$ & $4,146.0$ \\
Scalp & $1,805.1$ & $1,430.9$ & $1,556.0$ & $1,447.7$ & $1,718.9$ \\
Skin & $2,776.0$ & $2,552.9$ & $2,649.5$ & $2,602.5$ & $2,827.5$ \\
R eye & $1,183.2$ & 585.0 & 592.0 & 619.1 & $1,161.2$ \\
L eye & $1,145.9$ & 571.1 & 580.1 & 592.1 & $1,121.3$ \\
R optic nerve & $3,532.2$ & $2,630.8$ & $2,691.9$ & $2,644.3$ & $2,805.2$ \\
L optic nerve & $3,429.4$ & $2,645.2$ & $2,633.5$ & $2,443.2$ & $2,763.3$ \\
R lens & 483.5 & 346.3 & 369.1 & 366.7 & 736.0 \\
L lens & 477.3 & 350.9 & 366.7 & 360.6 & 705.4 \\
R internal auditory canal & $3,751.6$ & $3,512.5$ & $3,509.7$ & $3,392.1$ & $3,686.6$ \\
L internal auditory canal & $3,728.9$ & $3,466.2$ & $3,508.6$ & $3,427.6$ & $3,703.6$ \\
R external ear & $2,346.4$ & $2,020.8$ & $1,725.2$ & $1,423.7$ & $1,783.9$ \\
L external ear & $2,316.4$ & $1,945.4$ & $1,640.0$ & $1,306.8$ & $1,707.6$ \\
R cochlea & $3,652.9$ & $3,294.7$ & $3,301.6$ & $3,325.1$ & $3,435.4$ \\
L cochlea & $3,642.6$ & $3,252.2$ & $3,347.0$ & $3,269.6$ & $3,368.7$ \\
R parotid & $2,354.9$ & 905.6 & 917.8 & 773.2 & 966.6 \\
L parotid & $2,193.9$ & 820.9 & 816.7 & 731.1 & 955.5 \\
\hline
\end{tabular}

to the opposed lateral plans. Exceptions to improved dosimetric performance of the IMRT and VMAT plans were that VMAT increased the amount of low-dose radiation received by the scalp with an increase in $\mathrm{V}_{5}$ of $30 \%$ and a $\mathrm{V}_{10}$ increase of $10 \%$. The mean dose to the lens was also increased with VMAT (7.4 vs. 4.8 Gy OL).

On final analysis, of all the treatment planning techniques and potential beam arrangements, the 13-beam IMRT plan achieved the largest dose reduction for the scalp $\left(\mathrm{V}_{10}-10 \%\right.$, $\mathrm{V}_{20}-30 \%$, and $\left.\mathrm{V}_{30}-70 \%\right)$, external ear $\left(\mathrm{V}_{5}-10 \%\right.$, $\mathrm{V}_{10}-32 \%, \mathrm{~V}_{20}-28 \%$, and $\mathrm{V}_{30}-96 \%$ ), and parotid gland ( $-31 \%$ mean dose) combined.

\section{Discussion}

In this study, we constructed three IMRT plans and a singlearc VMAT plan for each patient studied to meet three

Table 4 Scalp dose-volume data according to treatment technique

\begin{tabular}{lcccc}
\hline & $\mathrm{V}_{5}(\%)$ & $\mathrm{V}_{10}(\%)$ & $\mathrm{V}_{20}(\%)$ & $\mathrm{V}_{30}(\%)$ \\
\hline 2-Beam OL & 67.6 & 64.0 & 54.3 & 28.0 \\
4-Beam IMRT & 62.5 & 54.8 & 40.0 & 13.2 \\
7-Beam IMRT & 69.7 & 62.6 & 44.0 & 12.0 \\
13-Beam IMRT & 65.6 & 57.2 & 40.1 & 7.6 \\
VMAT & 86.7 & 67.2 & 43.5 & 12.8 \\
\hline
\end{tabular}

$\mathrm{V} x$ volume receiving a specific dose $\left(\mathrm{V}_{5}=\right.$ volume receiving $\left.5 \mathrm{~Gy}\right)$ particular objectives: (1) deliver a homogeneous dose distribution to the PTV, (2) conformally reduce the dose delivered to the scalp, parotid glands, and external ears without sacrificing PTV coverage, and (3) ensure that other organs at risk did not receive more radiation dose than with traditional treatment planning using opposed lateral fields. Overall, we were able to accomplish these three objectives with each of the four plans that were created. As reported in the "Results" section of this paper, PTV coverage was maintained, and the mean dose to the PTV was improved with each of the

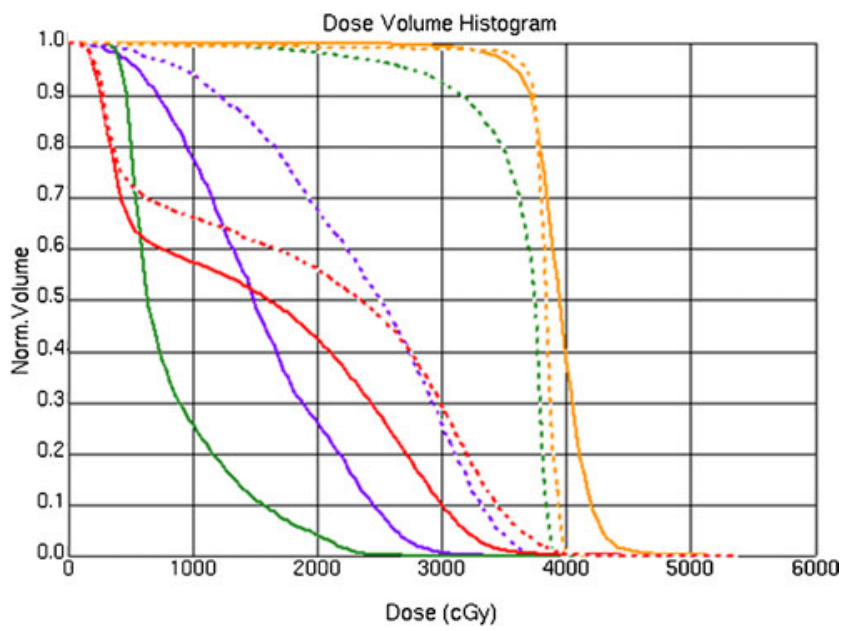

Fig. 2 This DVH represents a single, representative patient comparing 13-beam IMRT (solid lines) vs. standard OL (dashed lines). Structures include PTV (yellow), scalp (red), left parotid gland (purple), and left external ear (green) 
advanced treatment planning techniques. We observed that particular beam arrangements and planning techniques were suited differently depending upon the parameter being examined. For example, the four- and 13-field IMRT plans reduced dose to the scalp in all dose ranges $\left(\mathrm{V}_{5}, \mathrm{~V}_{10}, \mathrm{~V}_{20}\right.$, and $\mathrm{V}_{30}$ ), while the seven-field IMRT plan performed better with high dose levels $\left(\mathrm{V}_{20}\right.$ and $\left.\mathrm{V}_{30}\right)$ and the VMAT plan, although it would be superior in length of treatment time, increased projected delivery of low-dose radiation to the scalp. In future iterations of our volumetricmodulated arc planning technique for WBRT, we plan to use two arcs, rather than one, to allow a more even distribution of the dose and enable a decrease in the observed elevations of $V_{5}$ and $V_{10}$ doses to the scalp and mean dose to the lens (7.4 vs. $4.8 \mathrm{~Gy}$ ) seen for VMAT in this study.

Regarding the process of treatment planning and delivery of these plans, additional time on the part of the physician, dosimetry, and radiation therapy staff will be required to plan and deliver these advanced treatment plans. Creation of additional normal tissue contours, generating a clinical target volume (CTV)/PTV, the logistics of inverse treatment planning (part of which could be, however, scripted to improve efficiency), and actual radiotherapy delivery if a multiple-beam IMRT treatment plan is selected can all increase the amount of time spent per case. With respect to the normal tissue constraints that were originally placed on the organs at risk (Table 1), we found that it was extremely difficult to satisfy these parameters. We were, however, able to satisfy our original parotid gland, lens, and optic nerve constraints. It is possible that, with greater clinical experience using these techniques for WBRT, more achievable dose constraints and inverse planning parameters could be generated. Overall, the 13-beam IMRT plan had the largest dose reduction to those structures listed above. This is consistent with the fact that an increased number of beams promotes greater distribution of the dose and therefore has the potential to decrease the dose delivered to a particular structure. Of note, these dose reductions were accomplished without diminishing PTV coverage. These gains were not without sacrifice; however, in order to maintain PTV coverage and allow for a decrease in dose delivered to organs at risk, we had to permit an increased maximum dose delivered to the PTV. However, this increased maximum dose was delivered only to a small portion of the PTV and falls well below high-dose thresholds commonly used in the treatment of primary malignancies of the central nervous system or metastases using radiosurgery. Furthermore, because heterogeneity from IMRT may occur focally within the target volume, avoidance structures may be created for sensitive areas of the brain, including the hippocampus, with a limit for total dose included in the cost function for IMRT optimization. Final review of isodose distribution will be important with this treatment technique to ensure areas of maximum dose do not fall in unwanted regions of the brain.

Intensity-modulated treatment planning studies have been performed at other institutions primarily focusing on hippocampal avoidance [12] or dose reduction to the scalp [13-16], but to our knowledge, this is the first planning study that has combined several objectives into a single analysis. Our primary goal with testing this planning approach is to decrease dose delivery to surrounding normal tissue with the goal of decreasing or eliminating the noncognitive side effects of WBRT such as alopecia, xerostomia, and otitis. For scalp sparing IMRT, Roberge et al. reported a $53 \%$ measured dose reduction to the scalp while maintaining improving coverage to the CTV using an intensity-modulated approach [13]. Limmer et al. showed a similar reduction of mean dose to the scalp with a mean dose of $15 \mathrm{~Gy}$ and $D_{\max }$ of 25 Gy using tomotherapy [14]. Using a three-field technique to treat ten patients, Ting et al. were able to keep the mean scalp dose to 18.5 vs. 25 Gy for a conventional two-field plan and reported reduction in all volume-based dosimetric points $\left(\mathrm{V}_{90}, \mathrm{~V}_{80}\right.$, etc.) with only $50 \%$ of the patients experiencing "mild" hair loss [16]. With regard to hippocampal avoidance, it has been shown that dose reduction to the hippocampus using IMRT is possible while still maintaining appropriate coverage to the remainder of the brain [12]. Because nearly all brain metastases occur outside and away from the hippocampus [17], it is proposed that this region may be excluded in select cases of patients who present with no imaging evidence of metastasis near the hippocampus.

Concerns regarding the feasibility and/or potential use of IMRT or VMAT for the delivery of WBRT are primarily related to increased length of time required for treatment planning and delivery. With the increased number of beams and various couch angles required to deliver the radiation with four-, seven-, or 13-beam IMRT, treatment time would consequently increase. While increased length of treatment time was not specifically examined in our study, it is a very reasonable concern. We would propose that the potential application of IMRT/VMAT whole brain radiotherapy for brain metastases should be studied in a specific cohort of patients with longer than average anticipated life expectancy (either due to controlled systemic disease, favorable histology, and/or excellent performance status) $[4,18]$. This could also include patients treated in a prophylactic setting such as those with limited-stage or treatment-responsive extensive stage small cell carcinoma of the lung $[19,20]$. These patients would potentially have the longest length of time to benefit from reduced side effects from WBRT and could realize the most benefit in regard to enhanced quality of life. Although radiosurgery alone over combined treatment with WBRT has been shown to improve quality of life in at least one study [21], timely stereotactic radiosurgery is not available in all areas while centers that could offer intensity- 
modulated whole brain radiotherapy may be more readily available.

A limitation of our analysis and planning technique is that we were not able to reduce dose to all structures responsible for acute and chronic toxicity. To this end, one additional parameter that our clinic plans to improve upon is the radiotherapy dose delivered to the cochlea. While our IMRT and VMAT plans produced similar results to the opposed lateral plans in regard to cochlear dose (i.e., the dose to the cochlea did not increase with these planning techniques), further reduction of the cochlear dose may reduce or avoid hearing loss in patients. At the time of this publication, we have planned two cases in which the cochlea and internal auditory canal volumes were subtracted from the PTV within our intensitymodulated whole brain treatment plans. With this modification, dose delivered to the bilateral cochlea was reduced but, as expected, PTV coverage was somewhat compromised. Verifying that a patient has no micrometastases to the pericochlear region of the brain may allow exclusion of a small rim of tissue near the internal auditory meatus and cochlear avoidance more safe and feasible. An additional future step would be to include hippocampal avoidance into our plans with the intent of reducing both neurocognitive and noncognitive side effects. Hippocampal avoidance alone is presently being studied as a phase II trial by the RTOG, and we await results from their investigation. Finally, a needed next step will be to implement an institutional prospective, randomized clinical trial to evaluate the translation of improved DVH parameters as to whether they correlate with actual decreased acute and chronic toxicity observed in the clinic.

\section{Conclusion}

Intensity-modulated and volumetrically modulated arc treatment planning are capable of sparing dose to the scalp, parotid glands, and external auditory canal while maintaining comparable PTV coverage when compared to the traditional opposed lateral treatment technique. Such improvement is promising and may decrease noncognitive WBRT toxicities such as alopecia, xerostomia, and otitis, which could lead to an improved quality of life for patients diagnosed with multiple metastases to the brain.

Conflict of interest statement The authors declare that they have no conflict of interest.

\section{References}

1. Cairncross JG, Kim JH, Posner JB (1980) Radiation therapy for brain metastases. Ann Neurol 7:529-541
2. Posner JB, Chernik NL (1978) Intracranial metastasis from systemic cancer. Adv Neurol 19:575-587

3. National Cancer Care Network. NCCN Guidelines: central nervous system cancers (2011). http://www.nccn.org/professionals/ physician_gls/pdf/cns.pdf. Accessed 1 July 2011

4. Sperduto PW, Berkley B, Gaspar LE et al (2008) A new prognostic index and comparison to three other indices for patients with brain metastases: an analysis of 1,960 patients in the RTOG database. Int J Radiat Oncol Biol Phys 70:510 514

5. Patchell RA, Tibbs PA, Regine WF et al (1998) Postoperative radiotherapy in the treatment of single metastases to the brain: a randomized trial. JAMA 280:1485-1489

6. DeAngelis LM, Delattre JY, Posner JB (1989) Radiation-induced dementia in patients cured of brain metastases. Neurology 39:789796

7. Welzel G, Fleckenstein K, Schaefer J et al (2008) Memory function before and after whole brain radiotherapy in patients with and without brain metastases. Int J Radiat Oncol Biol Phys 72:13111318

8. Lawenda BD, Havaleh MG, Gierga DP et al (2004) Permanent alopecia after cranial irradiation: dose-reponse relationship. Int $\mathrm{J}$ Radiat Oncol Biol Phys 60:879-887

9. Tsao MN, Lloyd NS, Wong RKS et al (2005) Radiotherapeutic management of brain metastasis: a systemic review and metaanalysis. Cancer Treat Rev 31:256-273

10. Mehta MP, Tsao MN, Whelan TJ et al (2005) The American Society for Therapeutic Radiology and Oncology (ASTRO) evidence based review of the role of radiosurgery for brain metastasis. Int J Radiat Oncol Biol Phys 63:37-46

11. Gutierrez AN, Westerly DC, Wolfgang AT et al (2007) Whole brain radiotherapy with hippocampal avoidance and simultaneously integrated brain metastases boost: a planning study. Int J Radiat Oncol Biol Phys 69:589-597

12. Gondi V, Tolakanahalli R, Mehta MP et al (2010) Hippocampalsparing whole-brain radiotherapy: a "how-to" technique utilizing helical tomotherapy and LINAC-based intensity modulated radiotherapy. Int J Radiat Oncol Biol Phys 78(4):12441252

13. Yu JB, Shiao SL, Knisely JPS (2007) A dosimetric evaluation of conventional helmet field irradiation versus two-field intensitymodulated radiotherapy technique. Int J Radiat Oncol Biol Phys 68:621-631

14. Roberge D, Parker W, Niazi TM et al (2005) Treating the contents and not the container: dosimetric study of whole brain intensitymodulated radiation therapy. Technol Cancer Res Treat 4:567570

15. Limmer JP, Buskerd J, Henrich D, et al (2007) Scalp and ear sparing whole brain radiation therapy using helical tomotherapy. Poster session presented at: 49th Annual Meeting of the American Society of Radiation Oncology (ASTRO); Los Angeles, CA

16. Ting J, Thomas CR, McClure JA, et al (2005) Alopecia-less whole brain radiotherapy (WBRT) via IMRT: preliminary experience and outcomes. Poster session presented at: 47th Annual Meeting of the American Society of Radiation Oncology (ASTRO); Denver, $\mathrm{CO}$

17. Gondi V, Tome WA, Marsh J et al (2010) Estimated risk of perihippocampal disease progression after hippocampal avoidance during whole-brain radiotherapy: safety profile for RTOG 0933. Radiother Oncol 95:327-331

18. Gaspar L, Scott C, Rotman M et al (1997) Recursive partitioning analysis (RPA) of prognostic factors in three Radiation Therapy Oncology Group (RTOG) brain metastases trials. Int J Radiat Oncol Biol Phys 37:745-751 
19. Auperin A, Arriagada R, Pignon JP et al (1999) Prophylactic cranial irradiation for patients with small-cell lung cancer in complete remission. N Engl J Med 341:476-484

20. Slotman BJ, Mauer ME, Bottomley A et al (2009) Prophylactic cranial irradiation in extensive disease small-cell lung cancer: short-term health-related quality of life and patient reported symptoms: results of an international
Phase III randomized controlled trial by the EORTC Radiation Oncology and Lung Cancer Groups. J Clin Oncol 27(1):78-84

21. Chang EL, Wefel JS, Hess KR et al (2009) Neurocognition in patients with brain metastases treated with radiosurgery or radiosurgery plus whole-brain irradiation: a randomised controlled trial. Lancet Oncol 10(11):1037-1044 